\title{
SPECIFIC ALLERGEN IMMUNOTHERAPY RESULTS IN CHILDREN WITH ASTHMA DUE TO POLLENS ALLERGY. CLINICAL STUDY
}

\author{
Georgeta Sinitchi ${ }^{1}$, Aurica Rugina ${ }^{2}$, Smaranda Diaconescu ${ }^{2}$ \\ I "Apollonia” University, "Atopia” Allergological Medical Center, Iasi \\ 2 "Gr. T. Popa" University of Medicine and Pharmacology, \\ "Saint Mary” Clinical Emergency Pediatric Hospital, Iasi
}

\begin{abstract}
Introduction. Specific allergen immunotherapy (STI) is the causal treatment of asthma. SIT gives a specific response to the changing LT response (deviation of the immune response), induction of anergy (decrease of LTh2), induces tolerance and modifies the inflammatory response. Pollen produces in our country seasonal allergic asthma in children and adults.

Materials and methods. Our study was conducted using grass pollen extracts and comprised a total of 47 children with asthma, allergic to pollens, aged 5-14. Administration was seasonal (November to April), oral (sublingual solutions). It was indicated in the treatment of children with asthma and rhinitis with definite diagnosis where eviction was not possible. There have been previously detailed history and a careful clinical examination, skin prick tests to pollens with intense response, more elevated IgE, and spirometry confirming bronchospasm. Grass pollen extracts with initial low doses were used, gradually increasing the amount and concentration, and a gradual decrease, at a total of 6 months per year, with a duration of three years.

Discussion. The evaluation was performed by clinical follow-up (symptom score and drug consumption), spirometry and by improving the quality of life assessment. There were no serious side effects during treatment.

Conclusion. SIT in children with pollens allergic asthma is the causal therapeutic method; treating asthmatic children over 3 years old; reduces asthma symptoms to extinction; decreases other medical needs; improves quality of life of the child; no side effect were distinguished.
\end{abstract}

Keywords: child asthma, pollen allergy, specific immunotherapy

Specific allergen immunotherapy (SIT) is the causal treatment method of asthma, introduced by Noon and Freeman in the treatment of rhinitis and pollen induced allergic asthma. The techniques were established in 1918 by Cooke who also developed the hypo-thesis about the action mechanisms.

Administration ways were initially subcutaneous, and the sublingual way (oral mucosa) has proven its efficiency in 1990 and was recognized by World Health Organization (WHO) in 1998 and was used as pills since 2009 .

The use of anti IgE antibodies, capable of blocking the allergic fall, allows the immunotherapy potentiation by concomitant use of antiallergenic treatments.

The use of anti IgE antibodies will allow the decrease of allergenic immunotherapy side effects $(1,2)$.

New administration ways are being studied: intralymphatic (four shots in two months), subcuta- neously (3) using synthetic amino acids which would lead to a better tolerance, even long term, and a better efficiency (five shots), epicutaneously by applying allergic extracts on the scarification irritated skin.

The objectives of specific immunotherapy in allergic diseases, according to the European Medical Agency, highlighted by Alain Didier (4), were noted since 2008: 1 . The treatment of allergic symptoms, evaluated on short term and visible after a few months of treatment; 2. Maintaining the clinical effects evaluated through the efficiency persistency under treatment (more pollen seasons); 3. Long term effects correspond to the clinical efficiency persistency, after stopping the treatment for many more pollen seasons or more years and modifying the allergy's natural history; 4 . The last objective of immunotherapy is curing the allergic manifestations, characterized through the complete disap- 
pearance of the disease's symptoms as result of the treatment.

Specific immunotherapy in general, as the one in pollen allergies, modifies the allergic disease's natural history, reducing the appearance of new sensitivities and the risk of allergic rhinitis evolution to asthma $(5,6)$.

Pollen represents a series of particularities: seasonal production, variable quantities of day to day inhaled pollen and influences the clinical manifestations.

After 100 years of SIT practice, the "The European declaration of specific immunotherapy" (EAACI, June 13 2011) confirms the efficiency of this therapeutic method.

The mechanisms: specific allergen immunotherapy (SIT) is the causal treatment of asthma. SIT leads to a specific response, to changing the T Lymphocytes response (immune reaction deviation), anergy induction (decrease of LTh2), leads to tolerance and changes of the inflammatory reaction.
The oral immune system (7) has three subgroups of oral dendritic cells in mice and in humans, describes Langerhans cells in the oral mucosa and also preinflamatory cells in the sublingual region, vestibule and palate veil (8).

The oral immune system has also an organized lymphoid structure containing LB and LT adjusters and effectors.

Sublingual immunization with allergens that adheres and crosses the mucosa occurs in 15-30 minutes (Fig. 1).

The oral immune system induces immune tolerance through sublingual (oral) dendritic cells capable of LT ad-juster and LTh1 answer.

Sublingual specific immunotherapy decrease the LTh2 response, induces the increase of LTh1, decreases recruitment and activation of mastocytes, eosinophils, basophiles, increases seric IgG4; if initially the IgE levels increase, subsequent IgE seric response decreases, leading to increasing the level of IgA in the mucosas.

\section{Capture de l'allergène par les cellule dendritiques}

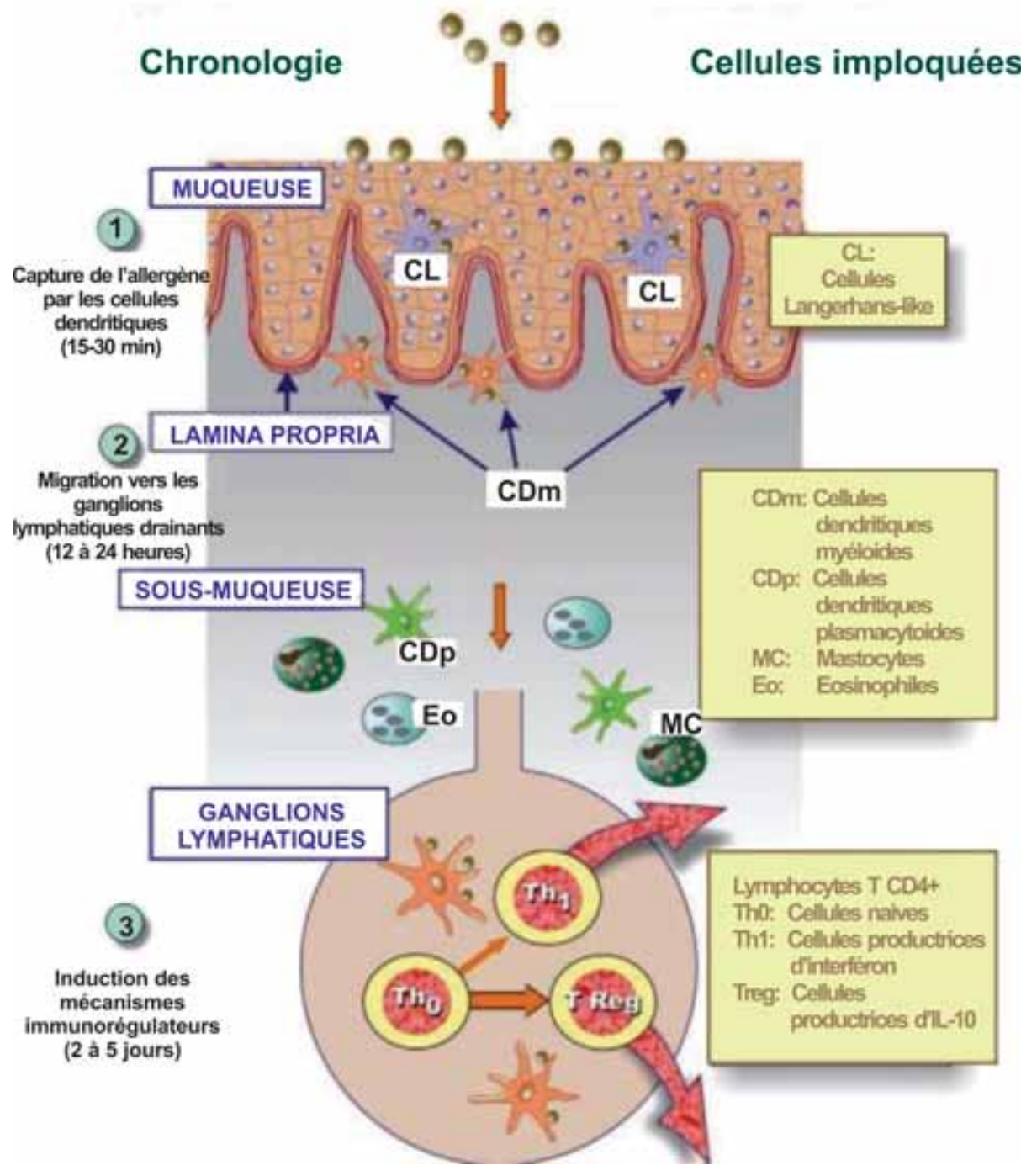

FIGURE 1. Sublingual imunisation - dendritic cells capturing allergens (Moingeon P., Mascarell L. [9]) 
Pollens produce in our country an allergic seasonal asthma in children and adults, and specific immunotherapy is done in preseason.

\section{MATERIAL AND METHODS}

Our study was made using Graminee pollen extracts on a number of 47 children.

The children had a close detailed medical history, with resumption in the discussion with them and the family, a thorough physical exam completed on a allergology type sheet.

Paraclinical examinations were prick test allergen determination and antibodies determination (IgE total and specific) in serum; there were also spirometry tests done.

The established immunotherapy was sublingual using Graminee pollens extract mixture, administrated preseasonal, twice per week, every three days, in small doses, progressively increasing the concentration and quantity, from the $1^{\text {st }}$ of November until April $-15^{\text {th }}$ of May for the duration of three years.

We did not include in the study children with oral cutaneous lesions, children in acute episodes or those less than five years of age.

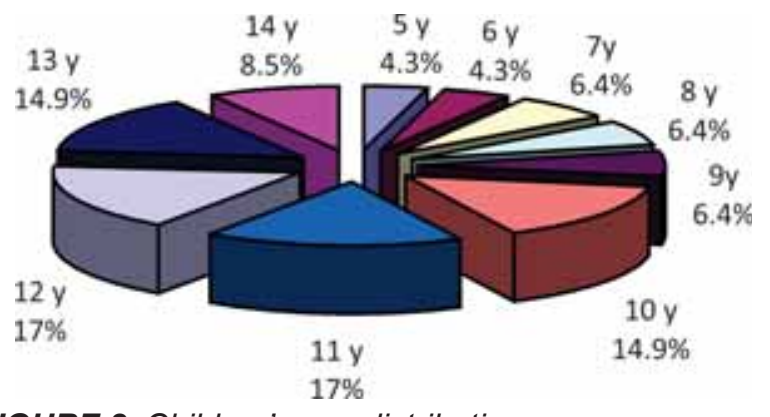

FIGURE 2. Children's age distributions

\section{RESULTS AND DISCUSSIONS}

Children included in the study were five to 14 years of age.

Asthma had seasonal manifestations, in the months of spring, summer, and late summer and the clinical form of intermittent asthma to moderate persistent asthma is represented in Fig. 3.

We mention that we did not have severe forms of asthma or uncontrolled asthma or disimmunitary diseases.

We had six monosensitized children, and the rest of 41 were multisensitized. The sensitivity intensities of cutaneous prick tests were very high especially to Graminee pollens. Polisensitized in our children study lot was according to Table 1 .

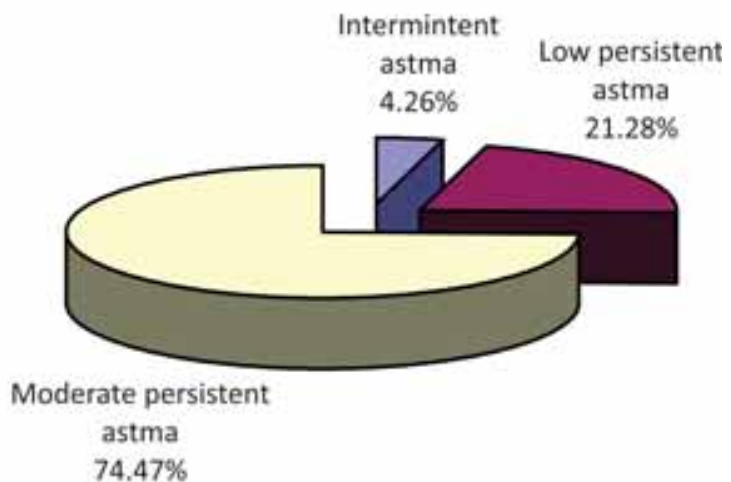

FIGURE 3. Clinical asthma on pollens allergic children included in the study.

TABLE 1. Polisensitization in pollens allergic children

\begin{tabular}{|l|c|l|c|}
\hline $\begin{array}{l}\text { Sensitizations } \\
\text { Environmental factors + } \\
\text { pollen }\end{array}$ & Nr. & $\begin{array}{l}\text { Sensitizations Food } \\
\text { factors + pollen }\end{array}$ & Nr. \\
\hline Mites & 29 & fish & 2 \\
\hline Molds & 14 & milk & 4 \\
\hline Dog hair & 3 & eggs & 6 \\
\hline Cat hair & 5 & celery & 3 \\
\hline Daphnia & 2 & soy & 4 \\
\hline & peanuts, nuts & 6 \\
\hline & seeds & 4 \\
\hline & raspberries & 3 \\
\hline & strawberries & 13 \\
\hline & &
\end{tabular}

Crossed allergies clinically mannifested through asthma crisis to pollen and food were present in nine children. The association of asthma, rhinitis and conjunctivitis was present in $99 \%$ of the cases.

There were no severe manifestations of anaphylactic shock. We appreciated the intensity of symptoms by symptomatic and clinical scores.

The first clinical reduced manifestations were of asthma crisis and rhinitis manifestations were reduced after.

The efficiency appreciation during the three years of study from April to September each year is illustrated in Fig. 4.

The association of respiratory manifestations with cutaneous was in proportion of $69 \%$.

The children's quality of life was appreciated through the symptoms score and the school attendance, increasing the quality of life.

The decrease of symptomatic therapeutic necessities was of $76 \%$.

Specific immunotherapy, from the economic point of view, leads to benefits because by reducing symptoms, it decreases the need for using symptomatic treatment according to literature data $(10,11)$ which we also found. Spirometry tests con- 


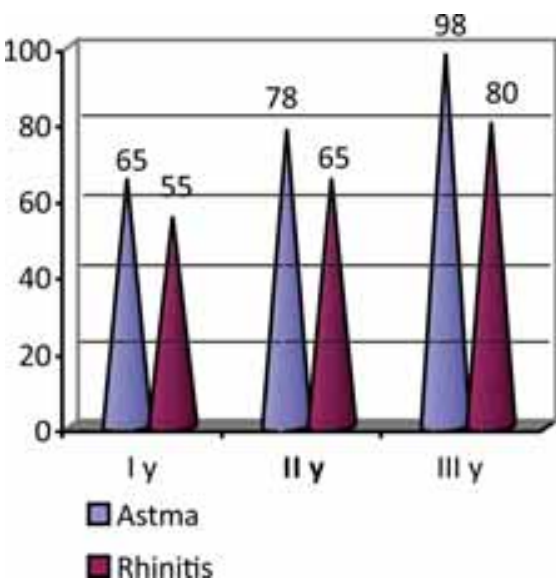

FIGURE 4. Efficiency of SIT during the study

firm the improvement through the reduction of bronchial spasm. We could have appreciated the results through immunologic determination, but we were unable to.

The SIT result appreciation can also be made through determinations of biomarkers, which can be correlated with the reductions of symptoms (12). One of the biomarkers is a specific antigen IgG4, next to LTh2 which decreases and modifies the values of interlokines 4 and 5 (IL4, IL5) (13).

SIT has, according to the ARIA 2001 consensus, antiinflammatory action: decreases the allergic inflammatory reaction (histamine, leukotrienes, prostaglandins, tryptase) and decreases target cells activation (mastocytes, basophils, eosinophils).

SIT has immunomulatory action: reduces the synthesis of LTh2 typed cytokines (IL4, IL13) synthesis of IgE and IL5 (airways inflammation), also stimulates the pro-Th1 antiinflammatory cytokines (IL12, IFN gamma and IL10).

SIT induces the rebalancing of LTh1-LTh2 and activates LTREG (14).

The immunomulatory action of SIT can be discussed considering the impact of the three dimensions of activation of $\mathrm{T}$ lymphocytes in allergic diseases, namely accentuating tolerance deficiency that is a real paradigm of atopic diseases (14) - Fig. 5.

Education for eviction measures and therapy was needed. We consider necessary the continuity of the treatment for another two years for a good result.

In our study we did not have adverse effects after sublingual SIT: oral pruritus, oral-labilial ede-
ANTIGEN-SPECIFIC IMMUNOTHERAPY ACTION MODE

The impact over the 3 dimensions of $T$ activation in allergic diseases

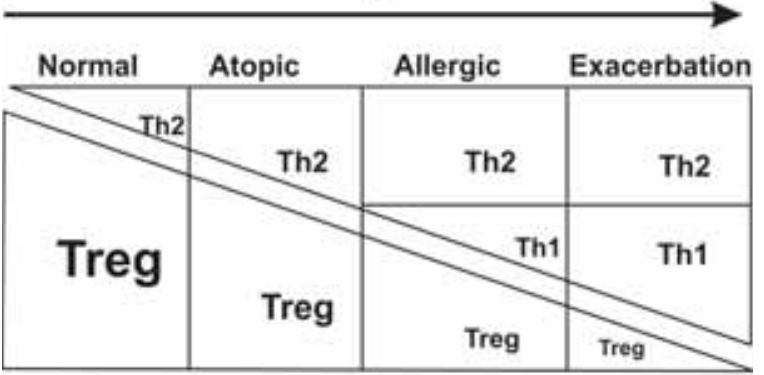

Is deficient tolerance the true paradigm for atopic diseases?

Magnan A., Humbert M. Clin Exp Allergy, 2005 Voir cours C. Ponvert

FIGURE 5. SIT Action mode

ma, nor rhinoconjunctivitis, digestive disorders, rashes, asthma or allergic shock.

The clinical efficiency appreciation, after the WHO 1998 and ARIA 2001-2007 consensus was:

- the decrease of rhinoconjunctivitis and asthma symptoms;

- the decrease of anti-M1 usage, inhalatory corticoids and bronchodilators;

- the decrease of nasal and specific bronchial hyperactivity.

\section{CONCLUSIONS}

- specific immunotherapy is the causal treatment for pollen allergic asthma;

- treatment was done sublingually, in preseason;

- clinical appreciation through symptomatic, drug and appreciation of the quality of life scores are necessary and confirm good results in high percentage;

- the compliance of the child and family is a positive factor of the therapy;

- the future of SIT will be molecular immunotherapy: treatment with new vaccines, obtained through the synthesis of oligonucleotides that code for recombination of allergens, coupled with compounds that increase immunogenicity, hybrid molecules and conjugated costimulation allergen-compounds. 


\section{REFERENCES}

1. Li J.T., Bernstein D.I., Calderon M.A., Casale T.B., Cox L., Passalacqua G., Pfaar O., Papadopoulos N.G.., Sublingual grass and ragweed immunotherapy: Clinical considerations-a PRACTALL consensus report, J Allergy Clin Immunol, pii: S0091-6749(15)010313. doi: 10.1016/j.jaci.2015.06.046, 2015;

2. Stokes J.R., Hartel R., Ford L.B., Casale T.B. Cannabis (hemp) positive skin tests and respira-tory symptoms, Ann Allergy Asthma Immunol, 85:238-40, 2000;

3. Senti, G. et al., Epicutaneous allergen administration as a novel method of allergen-specific immunotherapy, J. Allergy Clin. Immunol, 124, 997-1002, 2009;

4. Didier A., Malling H.J., Worm M., Horak F., Sussman G., Melac M., Soulié S., Zeldin R.K., Post-treatment efficacy of discontinuous treatment with 300 IR 5-grass pollen sublin-gual tablet in adults with grass pollen-induced allergic rhinoconjunctivitis. Clinical and experimental Allergy 2013 in press;

5. Dutau G., Immunothérapie spécifique, John Libbey, 2004

6. Rufin P., Désensibilisation aux pollens: présaisonnier ou cosaisonnier?, Revue française d'allergologie et d'immunologie Clinique 45, p. 555-560, 2005;

7. Mascarell L., Lombardi V., Zimmer A., Louise A., Tourdot S., L. Van Overtveltand P. Moingeon, Mapping of the lingual immune system reveals the presence of both regulatory and effector CD4 T cells, Clinical \& Experimental Allergy 39, 2009;

8. Moingeon P., et al., Novel routes for allergen immunotherapy: safety, efficacy and mode of action, Immunotherapy, 4(2):201-12, 2012;

9. Moingeon P., Mascarell L., Induction of tolerance via the sublingual route: mechanisms and applications, Clin. Dev. Immunol., 2011;

10. Donohue J., et al. Utilization and cost of immunotherapy for allergic asthma and rhinitis. Ann. All. Asthma and Imm. 82: 339-347, 1999;

11. Bucher K., Siepe M. Nutzen der hyposensibilisierung unter wirtschaftilchen aspekten, Aller-go J. Vol 4 3/95: 156-163, 1999;

12. Wachholz P.A., Durham S.R. Induction of 'blocking' IgG antibodies during immunotherapy. Clin Exp Allergy, 33:1171-4, 2003;

13. Fujimura Takashi, Yoshitaka Okamoto Antigen-Specific Immunotherapy against Allergic Rhinitis: The State of the Art, Allergology International, 59:21-31, DOI: 0.2332/allergolint.09RAI-0151, 2010;

14. Durham S.R. et al. Long-term clinical efficacy of grass-pollen immunotheraphy, The New Eng J of Med, 341:468-475, 1999;

15. Magnan A., Humbert M. Is deficient tolerance the true paradigm for atopic diseases?, Clin Exp Allergy, 2005 